This document is the unedited Author's version of a Submitted Work that was subsequently accepted for publication in 'Journal of the

American Society for Mass Spectrometry', copyright @ American Chemical Society after peer review. To access the final edited and published work see https://doi.org/10.1021/jasms.9b00043

\title{
How Hot are Your lons in Differential Mobility Spectrometry?
}

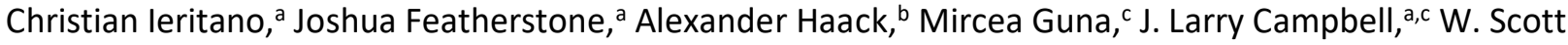
Hopkins $^{\text {a* }}$

${ }^{a}$ Department of Chemistry, University of Waterloo, 200 University Avenue West, Waterloo, Ontario, N2L 3G1, Canada. E-mail: shopkins@uwaterloo.ca

${ }^{b}$ Department of Physical and Theoretical Chemistry, University of Wuppertal, Gauss Str. 20, 42119, Wuppertal, Germany.

' SCIEX, 71 Four Valley Drive, Concord, Ontario, L4K 4V8, Concord, Ontario, Canada.

Keywords: Differential Mobility Spectrometry, Effective temperature, Thermometer ion, Benzylpyridinium 


\section{Abstract}

Ions can experience significant field-induced heating in a differential mobility cell. To investigate this phenomenon, the fragmentation of several para-substituted benzylpyridinium "thermometer" ions $(\mathrm{R}=\mathrm{OMe}, \mathrm{Me}, \mathrm{F}, \mathrm{Cl}, \mathrm{H}, \mathrm{CN}$ ) are monitored in a commercial differential mobility spectrometer (DMS). The internal energy of each benzylpyridinium derivative was characterized by monitoring the degree of fragmentation to obtain an effective temperature, $T_{\text {eff, }}$ which corresponds to a temperature consistent with treating the observed fragmentation ratio using a unimolecular dissociation rate weighted by a Boltzmann distribution at a temperature $T$. It was found that ions are sufficiently thermalized after initial activation from the ESI process to the temperature of the bath gas, $T_{\text {bath }}$. Once a critical field strength is surpassed, significant fragmentation of the benzylpyridinium ions was detected. At the maximum bath gas temperature $(450 \mathrm{~K})$ and separation voltage $(\mathrm{SV} ; 4400 \mathrm{~V})$ for our instrument, $T_{\text {eff }}$ for the benzylpyridinium derivatives ranged from $664 \pm 9 \mathrm{~K}(p-\mathrm{OMe})$ to $759 \pm 17 \mathrm{~K}(p-\mathrm{H})$. The extent of activation at a given SV depends on the ion's mass, degrees of freedom, $\left(N_{D o F}\right)$ and collision frequency as represented by the ion's collision cross section. Plots of $T_{\text {eff }}$ vs. the product of ion mass and $N_{D o F}$ and the inverse of collision cross section produce strong linear relationships. This provides an attractive avenue to estimate ion temperatures at a given SV using only intrinsic properties. Moreover, experimentally determined $T_{\text {eff }}$ correlate with theoretically predicted $T_{\text {eff }}$ using with a self-consistent method based on two-temperature theory. The various instrumental and external parameters that influence $T_{\text {eff }}$ are additionally discussed. 


\section{Introduction}

Ion mobility spectrometry (IMS) is a powerful tool for performing chemical analyses and separations. Whether operated as a standalone technique or coupled to mass spectrometry (MS), ion mobility measurements have been employed for structural elucidation in metabolomics, lipidomics, proteomics, and in the detection of illicit substances.[1-7] IMS separations are governed by the mobility of an analyte in a carrier gas while under the influence of an electric field, where ions navigate through the IMS cell in the field direction at velocity $v$ that depends on their mobility $K$ (Equation 1 ).

$$
v=K E=K_{0} N_{0} \cdot\left(\frac{E}{N}\right)
$$

In equation $1, K_{0}$ is the reduced ion mobility defined at standard temperature and pressure, $E$ is the electric field strength, and $N_{0}$ is the standard gas density. Depending on the technique, IMS instruments operate at different electric field strengths $(E)$ and gas number densities $(N)$. Under low $E / N$ conditions, thermal motion does not perturb ion drift velocities such that $K_{0}$ can be described by the Mason-Schamp relation (equation 2)[8]

$$
K=\frac{\sqrt{18 \pi}}{16} \sqrt{\frac{1}{m_{\text {ion }}}+\frac{1}{m_{\text {gas }}}} \frac{z e}{\sqrt{k_{b} T}} \frac{1}{\Omega \cdot \mathrm{N}}
$$

where $m_{\text {ion }}$ is the ion molecular mass, $m_{\text {gas }}$ is the molecular mass of the buffer gas, $z$ is the charge, $e$ is the elementary charge, $k_{b}$ is the Boltzmann constant, $T$ is the temperature, $N$ is the number density of the gas, and $\Omega$ is the ion-neutral collision cross section (CCS). Conventional drift-tube IMS (DTIMS) operates within the low field limit such that the Mason-Schamp equation is valid. Several commercial IMS techniques, such as traveling wave IMS (TWIMS) and differential mobility spectrometry (DMS), operate well above $E / N$ ratios that define the low-field limit $(c a .2-10 \mathrm{Td}),[5,9,10]$ which raises fundamental questions regarding ion transport due to the inherent dependence of ion mobility on field strength. 
The presence of a high electric field complicates the traditional Mason-Schamp approach to modelling ion transport, as the ion drift velocity is now significantly greater than its thermal velocity.[8, 11] In this highfield regime, no direct correlation can be established between ion drift time and CCS in temporally dispersive techniques. While this problem is circumvented in TWIMS analyses by empirically calibrating drift times of reference compounds against their known CCSs, no method to date can directly correlate an ion-neutral CCS to DMS parameters.[11]

The use of high electric fields raises further questions about whether an ion's native configuration is preserved, which has important implications for the interpretation of ion mobility data. It has been shown in both TWIMS and field-asymmetric waveform IMS (FAIMS),[12] an analog of DMS that uses cylindrical electrodes, that more elongated structures of proteins are observed compared to DTIMS.[13-15] Assessing the degree of field-induced ion heating is thus essential to interpret structural information from IMS experiments that operate in the high-field regime. However, gauging an ion's effective temperature also relies on determining the effect of the electric field on an ion's CCS.

For any collision between an ion and buffer gas within an IMS cell, the maximum energy that can be transferred between the collision partners is equal to the kinetic energy of the ion. Energy imparted to the ion through collision with the buffer gas is assumed to be equally partitioned amongst each degree of freedom. Equating the ion kinetic energy to the microcanonical kinetic energy described by statistical thermodynamics gives us an estimate of ion temperature as described by two-temperature theory (Equation 3)[8, 9, 16]

$$
T_{\text {eff }}=T_{\text {gas }}+T_{\text {field }} \approx T_{\text {gas }}+\frac{M}{3 k_{b}} v^{2}=T_{\text {gas }}+\frac{M}{3 k_{b}}(K E)^{2}
$$

where $T_{\text {gas }}$ is the temperature of the bath gas, $M$ is the mass of the bath gas, $v$ is the ion velocity, $K$ is the ion mobility, and $E$ is the electric field strength. As internal energy accumulates due to the frequency of collision, fragmentation and isomerization barriers can be overcome. In the case of benzylpyridinium (BP) 
thermometer ions, this is advantageous as the extent of fragmentation observed can be translated into an effective ion temperature with appropriate modelling of the thermally driven fragmentation kinetics. $[17,18]$. Temperature calibrations rely on an accurate determination of the bond dissociation energy ( $\mathrm{BDE}$ ) corresponding to the $\mathrm{C}-\mathrm{N}$ bond between the pyridine and benzylium moieties (Figure 1), which have been evaluated both experimentally[19, 20] and theoretically[19, 21-23] for numerous BP analogs. By monitoring the fragmentation of the parent ion, BP derivatives have been used to assess the internal energy of ions generated by electrospray ionization (ESI),[23-27] matrix-assisted laser desorption/ionization (MALDI),[28] and laser vaporization.[29] Analogous species including substituted benzylammonium ions[30] and more recently benzhydrylpyridinium ions[31] have been similarly used.

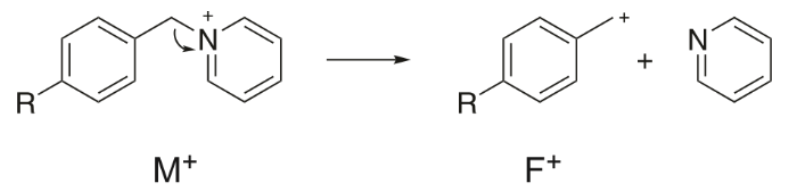

Figure 1. Fragmentation of benzylpyridinum thermometer ions $\left(\mathrm{M}^{+}\right)$to yield the substituted benzyl cation $\left(\mathrm{F}^{+}\right)$and pyridine.

For IMS, a quantitative measure of ion heating is essential owing to (1) the influence of temperature on CCS and (2) the distribution of conformers accessible at the effective ion temperature. In terms of TWIMS applications, Shvartsburg and Smith originally applied two temperature theory to estimate ion effective temperatures between 1600 to $7000 \mathrm{~K}$ depending on instrument parameters.[9] This was followed with two studies by Morsa et al., who determined effective ion temperatures using substituted BP ions on first and second generation TWIMS instruments (SYNAPT and SYNAPT G2) far below this initial estimate.[13, 32] As the effective ion temperature depends on the density of states of the analyte, temperatures were found to be $555 \mathrm{~K}$ in the first generation cell for the $p$-OMe BP derivative and between $629-751 \mathrm{~K}$ in the second generation TWIMS cell $(\mathrm{R}=p$-OMe, $p-\mathrm{F}, p-\mathrm{Cl}, p-t \mathrm{Bu})$. For larger thermometer ions, such as leucineenkephalin, Morsa et al. and Merenbloom et al. reported respective temperatures of $440 \mathrm{~K}$ and $449 \mathrm{~K}$ on 
the same second generation TWIMS cell.[13, 14] To build on the existing precedent for characterizing ion temperatures in IMS experiments, we present the use of a suite of para-substituted BP ions ( $R=$ OMe, $\mathrm{Me}, \mathrm{Cl}, \mathrm{F}, \mathrm{H}, \mathrm{CN}$ ) to characterize the effective temperature of ions in DMS for the first time. Prior to this work, only estimates of ion internal energy predicted by two-temperature theory and their relation to fragmentation efficiency were investigated.[33] This assessment is crucial to the development of DMS as a tool to evaluate an ion's CCS, as characterization of field-heating is vital to designing DMS workflows in which thermally driven structural rearrangements can affect data interpretation.

\section{Methods}

\section{Synthesis}

The respective para-substituted benzyl chloride derivative $(4.37 \mathrm{mmol})$ was dissolved in dry acetonitrile $(3 \mathrm{~mL})$ and treated dropwise with a dilute mixture of freshly distilled pyridine $(423 \mu \mathrm{L}, 5.24 \mathrm{mmol})$ in dry acetonitrile $(2 \mathrm{~mL})$ under nitrogen. The mixture was stirred overnight at room temperature, then subsequently concentrated by rotary evaporation. The product was precipitated using cold ether and isolated by filtration to afford the para-substituted benzylpyridinium chloride as a white powder $(40-68 \%$ yield).

Due to an isobaric contaminant in the mass spectrometer $(\mathrm{m} / \mathrm{z} 200)$, the $p$-OMe BP ion $(\mathrm{m} / \mathrm{z} 200)$ was prepared with pyridine- $d_{5}$ to generate the deuterated analog $(\mathrm{m} / \mathrm{z} 205)$. For simplicity, the deuterated species is referred to as $p$-OMe throughout the manuscript.

\section{Experimental Details}

A differential mobility spectrometer was mounted in the atmospheric region between the sampling orifice of a hybrid triple quadrupole linear ion trap mass spectrometer and electrospray ionization (ESI) source. This instrumental setup has been described elsewhere.[34-37] BP ions were introduced to the instrument 
by ESI at a flow rate of $10 \mu \mathrm{L} \mathrm{min}{ }^{-1}$. To prevent hydrolysis, BPs were solubilized in MeCN (200 ppb), then diluted to a working concentration of $10 \mathrm{ppb}$ in 9:1 MeOH:MeCN. The ESI probe was operated at $5500 \mathrm{~V}$ and ambient temperature using a nebulizing gas pressure of $20 \mathrm{psi}$ and an auxiliary gas pressure of 0 psi. These parameters were chosen to maximize ion production and minimize ion activation in the source. To minimize ion activation post-DMS while maintaining adequate signal, the following instrumental parameters were used by default unless stated otherwise: entrance potential (EP) of $2 \mathrm{~V}$, collision cell exit potential (CXP) of $0 \mathrm{~V}$, declustering potential (DP) of $0 \mathrm{~V}$, collision energy (CE) of $5 \mathrm{~V}$, DMS offset potential (DMO) of $-3 \mathrm{~V}$, collision gas (CAD) setting of low, and a curtain gas set to $20 \mathrm{psi}$. $\mathrm{N}_{2}$ was used as both the curtain gas (i.e., ESI desolvation gas and DMS carrier gas) and as the collision partner for collision induced dissociation (CID) studies (ca. 9 mTorr).

DMS experiments involved incremental stepping of the SV from 0 to $4500 \mathrm{~V}$. At each SV, ion intensity was monitored as the compensation voltage (CV) was scanned from $-5 \mathrm{~V}$ to $30 \mathrm{~V}$ in increments of $0.1 \mathrm{~V}$ to produce an ionogram. Data acquisition was completed using multiple reaction monitoring (MRM), where transitions corresponding to the $\mathrm{M}^{+} / \mathrm{M}^{+}, \mathrm{M}^{+} / \mathrm{F}^{+}$, and $\mathrm{F}^{+} / \mathrm{F}^{+}$were taken (see Figure 1 ). In our hybrid triplequad setup, an ion is selected in Q1, subjected to CID in Q2 (minimal collision energies; CE = $5 \mathrm{~V}$ ), and the fragment ions (or intact parent) are detected in Q3. For example, $\mathrm{M}^{+} / \mathrm{M}^{+}$corresponds to selecting the parent in Q1, allowing it to pass through the collision cell (Q2), and detecting the intact parent in Q3. This is done for all three MRM transitions, where each MRM transition has its own ionogram. lonograms corresponding to each MRM transition were integrated using the IntelliQuant package in Analyst 1.6.3. To account for ion focusing effects at high SV and CV, individual background-subtracted ionograms were normalized to the cumulative area of the three MRM transitions at each SV. Fragmentation yields (FY) and survival yields (SY) were calculated based on the peak areas (i.e. $A_{M+/ M+}, A_{M+/ F+}, A_{F+/ F+}$ ) of each normalized MRM transition (Equation 4). Determination of experimental SYs were conducted in triplicate for each BP ion. MRM transitions for each BP ion are reported in Table S1. 


$$
F Y=\frac{A_{F^{+} / F^{+}}}{\left(\left(A_{M^{+} / M^{+}}\right)+\left(A_{M^{+} / F^{+}}\right)+\left(A_{F^{+} / F^{+}}\right)\right)}=1-S Y
$$

To measure bath gas temperatures at various DMS cell temperatures, a J-type thermocouple was carefully placed between the two planar electrodes when all voltages on or near the DMS cell were set to $0 \mathrm{~V}$. This included the DMS electrodes, as well as the curtain plate voltage. These settings were zeroed, and safety interlocks were temporarily interrupted using software available to SCIEX research that is not commercially available.

\section{Modelling dissociation rates and effective temperatures}

The unimolecular rate constant is defined by Rice-Ramsperger-Kassel-Marcus (RRKM) theory (Equation 5) [38]

$$
k(E)=\frac{G^{\ddagger}\left(E-E_{0}\right)}{h \cdot \rho(E)}
$$

where $G^{\ddagger}\left(E-E_{0}\right)$ is the sum of states of the transition state that have an energy between zero and $E-E_{0}$, $\rho(E)$ is the density of states of the ground state, and $h$ is Planck's constant. The exact counts for the rovibrational sum and densities of states are evaluated using the Stein-Rabinovitch[39] extension of the Beyer-Swinehart[40] algorithm as implemented in MultiWell.[41-43] The transition states for heterolytic cleavage of the $\mathrm{C}-\mathrm{N}$ bond were treated as loose, which corresponds to a reaction pathway with no reverse activation barrier. Since the separated products that compose an orbiting transition state can freely rotate, the transition state sum of states is dependent on the angular momentum of the system. A reasonable limitation for these unimolecular dissociations is to treat the system in the phase space limit (PSL), where transitional modes in the transition state are assigned rotational constants equal to those of the separated products.[19, 44, 45] It should be noted that the structural isomerization from benzylium to tropylium occurs after initial dissociation.[46] For these calculations, it is convenient to approximate 
the BP parent and fragment ions as symmetric tops characterized by a $1 \mathrm{D}$ and two equal $2 \mathrm{D}$ rotational constants, which is defined as the geometric average of the most similar rotational constants, B and C, as $(B C)^{1 / 2}$. In the transition state, there is an additional $2 \mathrm{D}$ rotational constant that accounts for the angular momentum of the separating products. While this rotation is best treated variationally, the use of an effective rotational constant based on the pseudo-diatomic rigid rotor method described by Waage and Rabinovitch serves as a decent approximation.[47] All vibrational frequencies and rotational constants, including effective rotational constants associated with the external rotor, are provided in the supporting information.

Since RRKM rates are generated from theoretical BDEs and vibrational frequencies, error in the RRKM methodology $\left(\sigma_{\text {RRKM }}\right)$ is accounted for by scaling vibrational frequencies by $\pm 5 \%$ beyond the traditional scaling factors employed (vide infra). Additional scaling factors were applied as arrays of random numbers generated by random.org; six individual data sets for each BP derivative are modelled using the scaled vibrational frequencies, where the bond dissociation energy is also allowed to vary by the zero-point energy correction defined by the scaled frequency set. Each BP derivative was scaled by a different array of random numbers. RRKM rate coefficients for each BP derivative are shown in Figure S1.

To model effective ion temperatures in the DMS, a combination of RRKM theory and Boltzmann statistics is used. Since the collision rate, which occurs on the nanosecond timescale at atmospheric pressure, between an ion and neutral buffer gas is significantly greater than the ion transit time through the DMS cell, it is likely that ions are sufficiently thermalized and thus can be characterized by a Boltzmann distribution at temperature $T$ (Equation 6). This is a case where there is rapid energy exchange compared to dissociation; the dissociation rate, $k(T)$, can be described as a function of the microcanonical rate $k(E)$ from RRKM weighted by a Boltzmann energy distribution $\rho(E, T)$ at the temperature $T$ (Equation 7).[23] Thermal dissociation rates are shown in Figure S2. 


$$
\begin{gathered}
\rho(E, T)=\frac{\rho(E) \cdot \exp (-E / k T)}{\int_{0}^{\infty} \rho(E) \cdot \exp (-E / k T) d E} \\
k(T)=\int_{E_{0}}^{\infty} k(E) \cdot \rho(E, T)=\int_{E_{0}}^{\infty} \frac{G^{\ddagger}\left(E-E_{0}\right)}{h} \cdot \frac{\exp (-E / k T)}{\int_{0}^{\infty} \rho(E) \cdot \exp (-E / k T) d E} d E
\end{gathered}
$$

The probability of dissociation at a particular temperature, which is analogous to the observed FY, is given by Equation 8[19, 23]

$$
P_{d}(T)=F Y(T)=1-\exp (-k(T) \cdot \tau)
$$

where $\tau$ is the timescale for dissociation within the DMS cell. Given that $S Y=1-F Y$, the point at which the experimental SY matches that of Equation 8 for a BP ion is taken as the effective temperature, $T_{\text {eff. }}$.

As DMS is a spatially dispersive technique, residence time in the DMS cell $(\tau)$ is governed by the cell geometry and gas flow rate (Equation 9)

$$
\tau=\frac{V_{c e l l}}{Q}
$$

where $V_{\text {cell }}$ is the volume of the DMS cell and $Q$ is the volume flow rate. For a cell that is $3 \mathrm{~cm} \times 1 \mathrm{~cm} \times$ $0.1 \mathrm{~cm}$ and a baseline flowrate of $2.8 \mathrm{~L} \mathrm{~min}^{-1}\left(0.0467 \mathrm{~cm}^{3} \mathrm{~ms}^{-1}\right)$, the residence time is $6.43 \pm 1 \mathrm{~ms}$. With potential variability in the state of vacuum pumping, the error in the timescale for dissociation $\left(\sigma_{\tau}\right)$ is accounted for in the calculation of SY, and thus $T_{\text {eff. }}$

Due to the asymmetry about the inflection point, calculated survival yields are fit with a non-conventional 5-parameter logistic function ( $A_{\min }$ (minimum asymptote), $A_{\max }$ (maximum asymptote), $x_{0}$ (inflection point), h (Hill's slope), s (asymmetry factor); Equation 10), which is rearranged to correlate experimental SYs to an effective temperature $T_{\text {eff }}$ (Equation 11). Fits to calculated SYs are provided in Figure S3. All rate constants and fit parameters are provided in the supporting information.

$$
S Y_{\text {theor }}=A_{\text {min }}+\frac{\left(A_{\max }-A_{\min }\right)}{\left[1+\left(\frac{x_{o}}{T_{e f f}}\right)^{h}\right]^{s}}
$$




$$
T_{e f f}=x_{0}\left(\sqrt[s]{\frac{\left(A_{\min }-A_{\max }\right)}{\left(A_{\min }-S Y_{\text {expt }}\right)}-1}\right)^{-\frac{1}{h}}
$$

Absolute errors corresponding to determination of $T_{\text {eff }}$ originate from $\sigma_{\mathrm{RRKM}}, \sigma_{\tau}$, and from the reproducibility of experimental SYs $\left(\sigma_{S Y}\right)$. On the same day, SYs are reproducible to $1 \%(2 \sigma)$. However, three independent data acquisitions on different days resulting in an average deviation of $1.8 \%(2 \sigma)$ despite identical operating parameters. The greatest deviation in experimental SY was $3.5 \%$. Thus, $3.5 \%$ is used as the upper limit of uncertainty for the average SY of a single BP ion acquired in three independent data acquisitions (i.e. SYavg $(\%) \pm 3.5 \%$ ); error bars in $T_{\text {eff }}$ and correspond to the sum of $\sigma_{\mathrm{RRKM}}, \sigma_{\mathrm{\tau}}$ and $\sigma_{\mathrm{SY}}$.

\section{Computational Details}

All electronic structure calculations were performed using the Gaussian 16 B01 suite of programs.[48] Geometries of the intact benzylpyridinium ions and corresponding benzyl fragments were initially optimized at the B3LYP/6-31G level of theory, followed by subsequent optimization at the B3LYP/6$311++G(d, p)$ level of theory.[49, 50] DFT calculations included the GD3 empirical correction for dispersion.[51] Normal mode analyses were conducted to verify that each isomer corresponded to a minimum on the PES. Frequencies were scaled by 0.9679 to obtain the zero-point energy (ZPE).[52] Note that the scaling of vibrational frequencies by $\pm 5 \%$ for RRKM calculations is in addition to the initial scaling by 0.9679 . Atomic partial charges were generated according to the Merz-Singh-Kollman (MK) partition scheme, $[53,54]$ and constrained to reproduce the dipole moment of the BP derivative. All structures were carried forward to obtain theoretical CCSs in $\mathrm{N}_{2}$ using the MobCal-MPI package.[55] Single point energy calculations were performed on B3LYP/6-311++G(d,p) optimized geometries at the CCSD(T)/6$311++G(d, p)$ level of theory. Since experimental dissociation energies are only available for two of the BP derivatives used in this study,[19] calculated BDEs were employed for all molecules as a means of ensuring consistency. BDEs were calculated from $\operatorname{CCSD}(\mathrm{T})$ electronic energies and corrected with B3LYP/6- 
$311++G(d, p)$ ZPEs, which agree well with experimental values (Table S2). BDEs, geometries, and CCSs for each benzylpyridinium parent and fragment ion are provided in the supporting information.

\section{Results and Discussion}

\section{SV induced heating ( $p$-OMe)}

To characterize the extent of ion heating imparted by the SV waveform, we initially investigated ion heating due to interaction with the SV waveform for the most sensitive $p$-OMe BP derivative. Survival yields for the $p$-OMe BP ion as a function of the SV at different bath gas temperatures are shown in Figure 2A. As indicated by the SY of 0.65 at SV $=0 \mathrm{~V}$, significant ion activation occurs during the ESI process in our apparatus compared to other investigations.[30-32,56] It should be noted that the SY of the $p$ OMe BP ion remained unchanged until a critical SV (SV crit) was surpassed, which corresponds to a $>3.5 \%$ change in SY from the initial activation by ESI. At bath gas temperatures of 373,423 , and $450 \mathrm{~K}, \mathrm{SV}_{\text {crit }}$ values occur at $3750 \mathrm{~V}, 3250 \mathrm{~V}$, and $2750 \mathrm{~V}$, respectively. The observation of diminishing SV crit values as the bath gas temperature is increased while the initial SY remains unchanged supports the hypothesis that ions are thermalized to the temperature of the bath gas after the ESI process. 


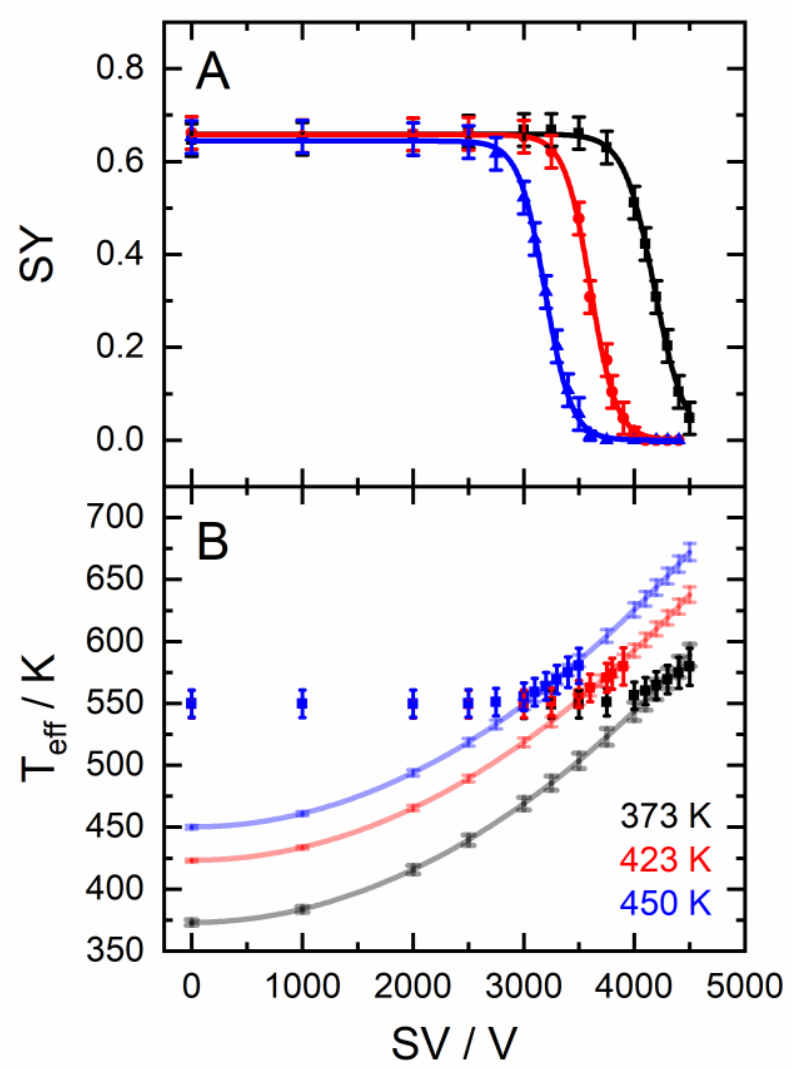

Figure 2. (A) p-OMe BP survival yields and (B) effective temperature at bath gas temperature of 373 (black), 423 (red) and $450 \mathrm{~K}$ (blue) are shown as solid squares. Curves in panel B are fits to $y=a x^{2}+b$ based on two temperature theory.

Figure $2 \mathrm{~B}$ shows the conversion of SY to $T_{\text {eff }}$ using RRKM modelling. In terms of correlating predicted $T_{\text {eff }}$ with those determined experimentally, the nature of the asymmetric SV waveform must be taken into consideration. The maximum voltage under the high-field condition is two-thirds of the peak-to-peak voltage applied (i.e., for SV $=3000 \mathrm{~V}, \mathrm{~V}_{\max }=2000 \mathrm{~V}$ ). In terms of $T_{\text {eff, }}$, ion-heating is at its maximum under the high-field condition, which therefore defines the fragmentation behavior of the ion for each SV cycle. Since the ions undergo approximately 20000 SV cycles during transit of the DMS cell, the observed $T_{\text {eff }}$ reflects the sum of $T_{\text {bath }}$ and the heating imparted during the high-field portion of the waveform. 
At $T_{\text {bath }}=373 \mathrm{~K}$, the $p$-OMe BP ion experiences a temperature of $561 \pm 8 \mathrm{~K}$ at an SV of $4200 \mathrm{~V}$. At higher bath gas temperatures at the same SV, the parent ion has already completely dissociated and RRKM underestimates the true $T_{\text {eff. }}$ With no observed change in SY before $\mathrm{SV}_{\text {crit }}$ or after complete dissociation, ion temperatures are approximated using a functional fit of $y=a x^{2}+b$. Effectively, $a$ becomes a collection of constants related to the ion being examined, and $b$ is the temperature of the bath gas. The fit is ultimately based on two-temperature theory, which follows a quadratic relationship with field strength (see equation 3). All fit parameters are available in the supporting information. However, the use of this quadratic fit is not optimal since the field dependence of mobility is not explicitly considered. When applying two-temperature theory, there are known deviations (ca. $10 \%$ ) at higher field strengths.[56] Although there are higher order corrections, as well as different approaches called momentum-transfer theory[57] and three-temperature theory, [58] to date there is no exact theoretical model that correctly predicts the mobility of an ion for high field strengths.

Like the deviations of the Mason-Schamp relationship at high field, the quadratic fit is expected to be more representative of ion temperature at lower field strengths. For example, consider $T_{\text {eff }}$ at a single SV across the three bath gas temperatures examined. The difference in effective temperatures should be approximately equal to the difference between bath gas temperatures with an additional contribution from the dependence of ion mobility $K$ on temperature. A plot of the differences in RRKM and fit temperatures are shown in Figure $\mathrm{S} 4$. Considering $T_{\text {bath }}$ conditions of $373 \mathrm{~K}$ and $423 \mathrm{~K}$, one expects a temperature difference of $\Delta T_{\text {bath }}=50 \mathrm{~K}$; it was found that that the average difference between effective ion temperatures is $\Delta T_{\text {eff }}=49 \pm 5 \mathrm{~K}$. Similarly, for $\Delta T_{\text {bath }}=77 \mathrm{~K}$ and $\Delta T_{\text {bath }}=27 \mathrm{~K}, \Delta T_{\text {eff }}$ was found to be 80 $\pm 5 \mathrm{~K}$ and $\Delta T_{\text {eff }}=32 \pm 4 \mathrm{~K}$, respectively. Given that differences in effective temperature correspond to the differences in bath gas temperature, one can use a quadratic fit to estimate ion temperatures before $\mathrm{SV}_{\text {crit, }}$ and after the parent ion has completely fragmented. For example, at $\mathrm{SV}=3200 \mathrm{~V}$, the $p$-OMe BP ion experiences effective temperatures of $482 \pm 6 \mathrm{~K}, 532 \pm 4 \mathrm{~K}$, and $564 \pm 11 \mathrm{~K}$ at the three bath gas 
temperature settings. The fit provides decent approximations for ion effective temperatures prior to $\mathrm{SV}_{\text {crit }}$ but will likely produce poor approximations for ion temperature beyond the range that the RRKM-based method employed here can probe (i.e., when then parent has completely fragmented). Thus, alternative $\mathrm{BP}$ ions with dissociation thresholds greater than that of $p$-OMe BP were investigated.

\section{SV induced heating $(\mathrm{R}=\mathrm{Me}, \mathrm{F}, \mathrm{Cl}, \mathrm{H})$}

Figure 3 shows the SY of various BP ions $(\mathrm{R}=\mathrm{Me}, \mathrm{F}, \mathrm{Cl}, \mathrm{H})$ as a function of the SV. Due to the higher energy required for dissociation of these species, fragmentation was only observed when using bath gas temperatures of $450 \mathrm{~K} . \mathrm{SV}_{\text {crit }}$ increases as the dissociation energy corresponding to loss of pyridine increases for the various of BP derivatives. It should be noted that the $p$-CN BP derivative (not shown in Figure 3) did not exhibit any degree of additional fragmentation in the DMS cell due to its high $E_{0}$.

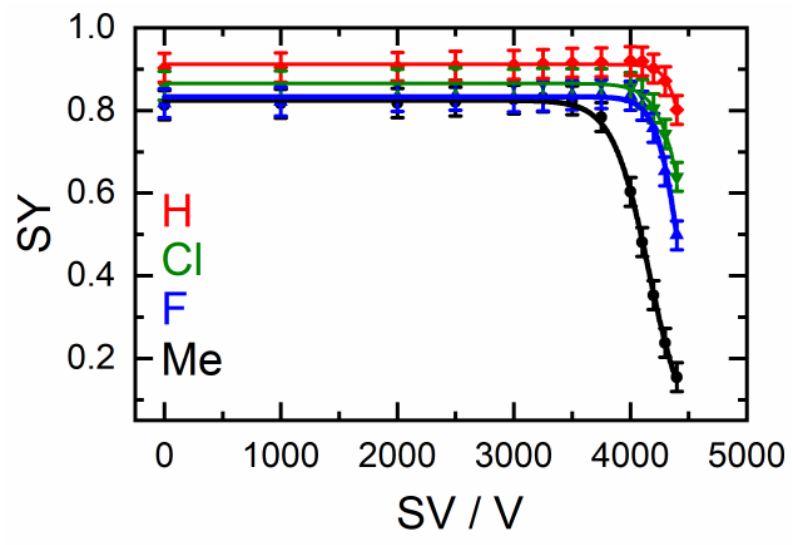

Figure 3. Survival yields for the para substituted benzylpyridinium ions $(\mathrm{R}=\mathrm{H}, \mathrm{Cl}, \mathrm{F}, \mathrm{Me})$ at $T_{\text {bath }}=450 \mathrm{~K}$.

In contrast to the $p$-OMe BP ion, the survival yields of the other derivatives at SV $=0$ are not significantly reduced. Again, only in-source fragmentation for the BP derivatives is observed before $S V_{\text {crit }}$ is surpassed, indicating efficient thermalization of the analytes. The $p$-Me BP derivative $\left(E_{0}=2.27 \mathrm{eV}\right)$ exhibits $\mathrm{SV}_{\text {crit }}=3800 \mathrm{~V}$, whereas the $p-\mathrm{H}$ BP derivative $\left(\mathrm{E}_{0}=2.50 \mathrm{eV}\right)$ exhibits $\mathrm{SV}_{\text {crit }}=4300 \mathrm{~V}$. Despite these high 
values of $S V_{\text {cruit, }}$ one can correlate the observed SYs to temperatures with RRKM modelling and quadratic fits. The result of this analysis is shown in Figure 4.

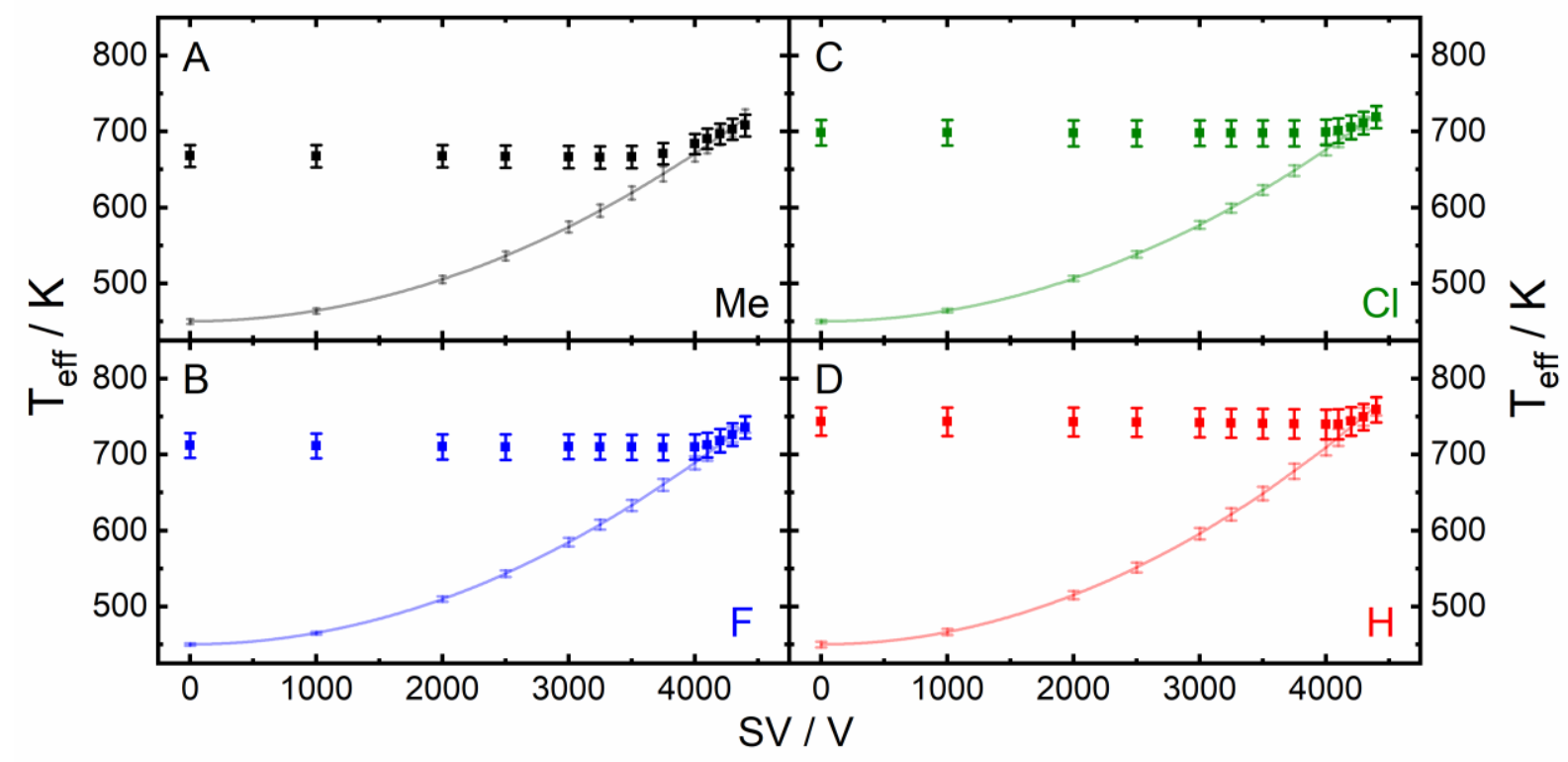

Figure 4. Effective temperatures for the $p-\mathrm{Me}(\mathbf{A}), p-\mathrm{F}(\mathbf{B}), p-\mathrm{Cl}(\mathbf{C})$, and $p-\mathrm{H}(\mathbf{D})$ benzylpyridinium derivatives at $T_{\text {bath }}=450 \mathrm{~K}$ are shown as solid squares. Curves are fits to $y=a x^{2}+b$ based on two temperature theory.

The separation field in the DMS cell (gap width of $1 \mathrm{~mm}$ ) cannot be increased ad infinitum due to the breakdown voltage of the carrier gas. At the breakdown point, the buffer gas becomes electrically conductive and results in arcing between the stainless steel DMS electrodes. The $T_{\text {eff }}$ for the BP derivative ions at the maximum stable SV (4400 V) are summarized in Table 1 . The unique $T_{\text {eff }}$ experienced by each BP ion at a given SV ultimately stems from its inherent ability to partition internal energy throughout its various (unique) internal degrees of freedom.

\section{The Dependence of $T_{\text {eff }}$ on Ion Structure}

To explore the $T_{\text {eff }}$ dependence on analyte structure (viz. geometry and internal energy), $T_{\text {eff }}$ was evaluated for the five BP derivatives that exhibited field-induced fragmentation at a $T_{\text {bath }}=450 \mathrm{~K}$. Table 1 summarizes 
the properties that influence the values of $\mathrm{T}_{\text {eff. }}$. These properties include the ion mass $m$, the total degrees of freedom $N_{D o F}, E_{0}$, and the collision cross section in $\mathrm{N}_{2}\left(\Omega_{\mathrm{N} 2}\right)$.

Table 1. Properties of BP ions and their corresponding $T_{\text {eff }}$ at $\mathrm{SV}=4400 \mathrm{~V}\left(T_{\text {bath }}=450 \mathrm{~K}\right)$.

\begin{tabular}{|c|c|c|c|c|c|}
\hline Ion & $T_{\text {eff }} / \mathrm{K}$ & $m / z$ & $\mathrm{~N}_{\text {DoF }}$ & $\Omega_{\mathrm{N} 2} / \AA^{2}$ & $\mathrm{E}_{0} / \mathrm{eV}$ \\
\hline$p-\mathrm{OMe}$ & $664 \pm 9 \mathrm{~K}^{\mathrm{a}}$ & 205.14 & 87 & 143.09 & 1.85 \\
\hline$p-\mathrm{Me}$ & $708 \pm 14 \mathrm{~K}$ & 184.11 & 84 & 139.54 & 2.27 \\
\hline$p-\mathrm{F}$ & $736 \pm 15 \mathrm{~K}$ & 188.09 & 75 & 136.19 & 2.39 \\
\hline$p-\mathrm{Cl}$ & $719 \pm 15 \mathrm{~K}$ & 204.06 & 75 & 141.28 & 2.37 \\
\hline$p-\mathrm{H}$ & $759 \pm 17 \mathrm{~K}$ & 170.10 & 75 & 133.51 & 2.50 \\
\hline$p-\mathrm{CN}$ & $\mathrm{n} / \mathrm{a}$ & 195.09 & 78 & 149.55 & 2.74 \\
\hline
\end{tabular}

${ }^{a} T_{\text {eff }}$ determined with a quadratic fit (see blue trace, Figure $2 \mathrm{~B}$ ) due to complete fragmentation of parent.

The total energy imparted to an ion is related to its collision frequency with the buffer gas, which is proportional to its CCS; larger molecules traveling at the same velocity as smaller molecules gain more internal energy. However, since geometrically larger species have lower mobilities in a given collision environment, larger molecules travel more slowly than smaller molecules of equal mass and exhibit lower kinetic energy, and therefore lower $\mathrm{T}_{\text {eff. }}$ In other words, ion internal energies in the DMS environment are governed by two opposing effects; ion internal energies increase with ion CCS, but ion effective temperatures are diminished by lower mobilities with increasing ion CCS.

Under thermalization conditions, the $\mathrm{T}_{\text {eff }}$ of a molecule describes its kinetic and internal energy, which is partitioned among its translation and internal (i.e., rotational and vibrational; electronic ground state is assumed) degrees of freedom. Bearing this in mind, $T_{\text {eff }}$ of a BP derivative is proportional to its density of states and collision frequency. To a first approximation, the density of states of a molecule can be described by its mass-weighted degrees of freedom. Figure 5 illustrates the relationship between $T_{\text {eff }}$ of the five BP derivatives investigated at $T_{\text {bath }}=450 \mathrm{~K}$ and the product of the ion mass, its $3 \mathrm{~N}$ degrees of freedom, and the inverse of its CCS. Linear fit parameters for heating at all SVs sampled can be found in 
the supporting information. At the same SV, smaller ions (i.e., those with lower mass and $N_{D o F}$ ) exhibit greater $T_{\text {eff }}$ compared to larger ions. This approach follows observations made by Donor and coworkers, who noted the efficiency of energy deposition in proteins due to collisional activation was proportional to their mass and kinetic energy.[59] While the observed linear trend provides an attractive avenue to estimate effective ion temperature at a given SV using only intrinsic ion properties, more work must be done to validate the generality of the approach.

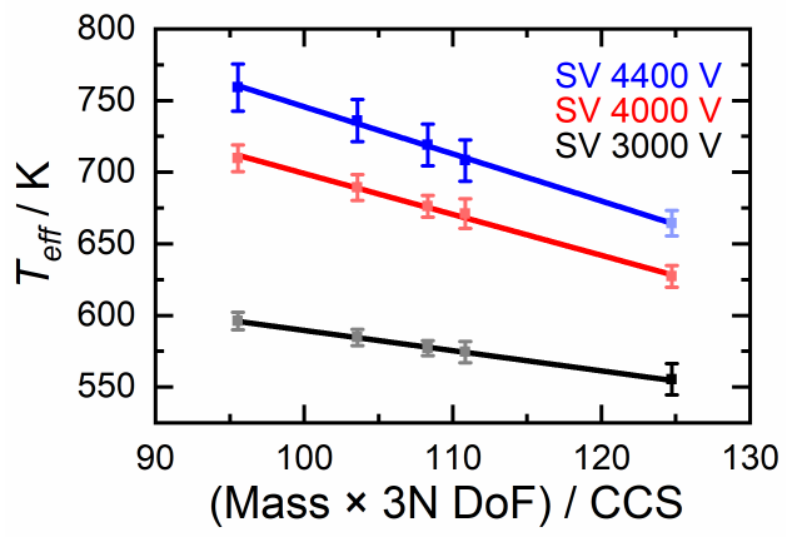

Figure 5. Correlation of $T_{\text {eff }}$ determined at $S V=4400 \mathrm{~V}$ (blue; $\left.\mathrm{R}^{2}=0.998\right), 4000 \mathrm{~V}$ (red; $\mathrm{R}^{2}=0.996$ ), and $3000 \mathrm{~V}$ (black; $\left.\mathrm{R}^{2}=0.998\right)$ with ion mass, $3 \mathrm{~N}$ degrees of freedom, and inverse CCS $\left(T_{\text {bath }}=450 \mathrm{~K}\right)$. Darker squares indicate $T_{\text {eff }}$ values determined from the conversion of experimental SYs using the RRKM method; lighter squares indicate extrapolated $T_{\text {eff }}$ determined from fits to $y=a x^{2}+b$ as shown in Figures $2 \mathrm{~B}$ and 4B.

\section{Two-temperature Theory Predications of $T_{\text {eff }}$}

Using the principles of two-temperature theory, it is possible to model the $T_{\text {eff }}$ of an ion under the dynamic conditions of the DMS environment.[60] Briefly, the drift velocity of each BP derivative was evaluated using Equation 1, where ion mobility $K$ is defined by the Mason-Schamp relation (Equation 2). $K$ was 
evaluated using temperature-dependant CCS calculations performed in MobCal-MPI. The CCSs were fit to a function of the form $a+b\left(T_{\text {eff }}\right)^{c}$, which is based on the proportionality of CCS to $T^{-1 / 2}$ in the low-field limit. The physical interpretation of the offset $a$ corresponds to the CCS in the infinite temperature limit.[61] It should be noted that $\Omega_{N 2}(T)$ deviates from the inverse square-root dependence at higher $T$, which likely originates from deviations in Mason-Schamp predictions at high temperature. The variable exponent $c$ allows for a more accurate interpolation, which is a viable alternative compared to calculation of the CCS at each temperature iteration in the self-consistent approach. The corresponding $T_{\text {eff }}$ and ion velocities determined by this technique are shown in Figure $\mathrm{S} 5$ at $T_{\text {bath }}=450 \mathrm{~K}$.

Owing to the nature of the SV waveform as described previously, a comparison must be made between experimental $T_{\text {eff }}$ and the value predicted by two-temperature theory during the high-field portion of the waveform where ion heating is maximized. This comparison is shown for the $p$-OMe (lowest error) and $p$ $\mathrm{Cl}$ (biggest error) in Figure 6 for $T_{\text {bath }}=450 \mathrm{~K}$. Similar correlation plots for the other derivatives are provided in Figure S6. 


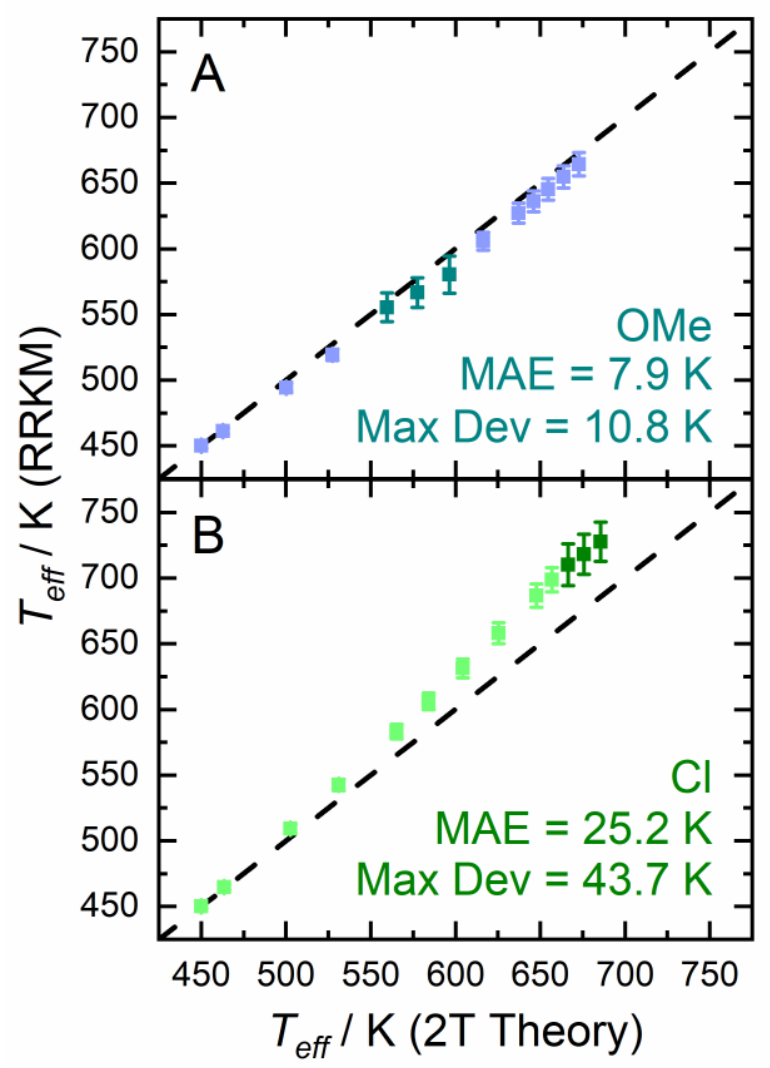

Figure 6. A comparison of experimental and theoretical $T_{\text {eff }}$ determined using a self-consistent two temperature theory approach for the (A) $p$-OMe and (B) $p$-Cl BP derivatives. The dashed line corresponds to $y=x$. Lighter squares indicate extrapolated $T_{\text {eff }}$ determined from fits to $y=a x^{2}+b$ as shown in Figures 2B and 4. Darker squares indicate $T_{\text {eff }}$ values determined from the conversion of experimental SYs using the RRKM method.

Overall, there is good agreement between $T_{\text {eff }}$ determined experimentally and that calculated by twotemperature theory. Mean absolute errors (MAE) between the two methods range from 7.9 K ( $p$-OMe BP) to $25.2 \mathrm{~K}(p-\mathrm{Cl})$. Deviations are systematic in that two-temperature theory underestimates ion temperature at high $T_{\text {eff }}$ (high SV). These deviations can be attributed to: (1) the field dependency of ion mobility $K$ above the low field limit, (2) neglecting instantaneous ion velocities (and therefore dynamic temperature variations) resulting from the dynamic waveform, and (3) the possibility of ion heating during 
the low field portion of the waveform at high SV. To address these points in more detail, deviations at high $T_{\text {eff }}$ can be largely attributed to the breakdown of the Mason-Schamp relation at high-field. This is best treated empirically with an $\alpha$ function (Equation 12), where the $\alpha$ coefficients define the difference in ion mobility between high and low field. Since reversing the field polarity has no impact on ion mobility, the series expansion is a summation of even powers.

$$
K\left(\frac{E}{N}\right)=K(0) \cdot\left[1+\alpha\left(\frac{E}{N}\right)\right]=K(0) \cdot\left[1+\alpha_{2}\left(\frac{E}{N}\right)^{2}+\alpha_{4}\left(\frac{E}{N}\right)^{4}+\cdots\right]
$$

Since all BP ions require increasingly positive CVs to elute as SV increases (Type $C$ behavior), $\alpha$ coefficients become increasingly negative as field strength increases.[35, 62] This describes a reduced ion mobility under high field conditions compared to low-field, which results in reduced ion velocities and a comparatively lower $T_{\text {eff. }}$ While $\alpha$ coefficients are calculated and employed in this approach, one must also consider the shape of the SV waveform and its effect on instantaneous ion velocities. For the implementation studied here, the combination of two sinusoidal harmonics means ions experience the peak potential of the waveform for a small fraction of the duty cycle. DMS trajectory simulations show that ions adopt zig-zag-like paths reflecting the SV waveform, but with variation due to diffusion.[63] Ultimately, this results in instantaneous velocities of zero during the ion's transit due to the switching SV polarity and trajectory fluctuations associated with ion diffusion. Consequently, ions are unlikely to reach their steady-state drift velocity KE during the high or low field portion of the SV waveform. Simply put, ions do not instantaneously adjust to the dynamic field, leading to the inequality $v \leq K E$, which will be more pronounced at higher field strengths. One would therefore expect that measured ion temperatures should be lower than those predicted by two-temperature theory. However, this is offset by the fact that a non-negligible degree of ion fragmentation occurs across the entire SV duty cycle (not just at the highest point of the waveform), and this behavior is not captured in two-temperature theory modeling. Thus, the ideal method to predict $T_{\text {eff }}$ using a first-principles two-temperature theory approach would involve 
simulating ion trajectories in the DMS cell to evaluate instantaneous and temperatures across the entire SV duty cycle. A basis for this approach was first described by Lai and coworkers, who simulated ion mobility in $\mathrm{N}_{2}$ and $\mathrm{He}$ at high field strengths. [64] Coupling of these simulations with the inelasticity of collisions and ion diffusion would allow for evaluation of ion velocities over numerous SV duty cycles. These velocities could then be used in a self-consistent approach to evaluate $T_{\text {eff; }}$ our efforts in this regard are ongoing.

\section{The additional effects of instrumental parameters on ion heating}

\section{Curtain gas flow}

The gas number density $N$ is directly proportional to the gas pressure. Given the inherent increase in collision frequency at higher operating gas pressure, one would expect a decrease in ion $T_{\text {eff }}$ due to the reduced $E / N$ ratio. Recall that the time between collisions in DMS experiments at atmospheric pressures is on the nanosecond timescale. Over a residence time of $6.4 \pm 1 \mathrm{~ms}$, during which millions of collisions occur between the ions and the background gas, it is expected that ions thermalize efficiently. Since a change in curtain gas pressure is expected to have only a minor effect on the total pressure in the DMS cell, its anticipated collisional cooling effect should be small. This is observed experimentally, where the most drastic SY differential over the $10-30$ psi range (at $\mathrm{SV}=4400 \mathrm{~V}$ ) is $\Delta \mathrm{SY}=0.16 \pm 0.05$ for the $p-\mathrm{Cl} \mathrm{BP}$ derivative. For the $p-\mathrm{H}, p-\mathrm{F}$, and $p$-Me derivatives, $\Delta \mathrm{SY} \approx 0.11 \pm 0.05$ for the same conditions, and $\Delta S Y=0.08 \pm 0.05$ for the $p$-OMe derivative at $S V=3200 \mathrm{~V}$ (above which ion signal is lost due to significant fragmentation of the parent). These results are summarized in Figure S7. Should one desire to tune $T_{\text {eff }}$ by way of changing curtain gas pressure, one should be cognisant of the fact that higher pressures result in reduced ion transmission through the DMS cell. 


\section{Ion optics voltages; entrance potential (EP) and collision cell exit potential (CXP)}

To explore the field-induced heating effect of the ion optics voltages, parameters were first set to minimize ion activation (i.e., $\mathrm{EP}=2 \mathrm{~V}, \mathrm{CXP}=0 \mathrm{~V}, \mathrm{DMO}=-3 \mathrm{~V}, \mathrm{DP}=0 \mathrm{~V}$ ), then individual parameters were varied. In this way, the effect of post-DMS potentials on ion heating was investigated by conducting SY evaluations at $T_{\text {bath }}=373 \mathrm{~K}$ for the most sensitive BP derivative ( $p$-OMe). The results of these studies are shown in Figure S8. In terms of the EP and CXP, there is very little difference in SY between operating conditions that minimize ion activation and those that optimize ion transmission. However, it should be noted that the effect of field focusing is more pronounced at the optimal experimental settings and could impact SY evaluations if the efficiency of ion transmission is different for the parent and fragment ions. Thus, it is recommended to characterize $T_{\text {eff }}$ under conditions that minimize ion activation to mitigate some of the effects arising from field focusing. Operating under default conditions (i.e., EP = $10 \mathrm{~V}, \mathrm{CXP}=$ $15 \mathrm{~V}, \mathrm{DMO}=-3 \mathrm{~V}, \mathrm{DP}=100 \mathrm{~V}$ ) significantly increases $T_{\text {eff }}$ at $\mathrm{SV}=0 \mathrm{~V}$ from $550 \pm 11 \mathrm{~K}$ to $564 \pm 11 \mathrm{~K}$. This increase in effective ion temperature is largely due to fragmentation induced by the DP. At DP $=100 \mathrm{~V}$, $T_{\text {eff }}$ at SV $=0$ is $561 \pm 11 \mathrm{~K}$, indicating that the EP and CXP collectively contribute $c a .3 \mathrm{~K}$ of heating to the p-OMe BP ion.

\section{Declustering Potential (DP)}

The DP setting controls the voltage on the orifice between the DMS cell and MS, which is used to decluster ions prior to entry into the QJet region that focuses ions into Q1. Since the pressure in the QJet region is held at approximately 2 Torr, E/N ratios exceed $150 \mathrm{Td}$ at $\mathrm{DP}=100 \mathrm{~V}$ (see figure S9). The high field in this region induces additional fragmentation of the benzyl cation $\left(\mathrm{F}^{+}\right)$fragments beyond the unimolecular dissociation leading to loss of pyridine (see Figure 1). Since the modelling of these additional fragmentation pathways with RRKM requires characterization of the (unknown) transition states, a traditional SY method described previously was instead employed to estimate $T_{\text {eff. }}[23,25,26,28,65]$ 
Rather than solely monitoring the substituted benzyl fragment cation, this method instead accounts for all fragments.[19, 66] Figures S10 to S15 plot the observed fragmentation channels as breakdown curves.

This alternative method plots SY values against calculated BDEs for $\mathrm{C}-\mathrm{N}$ cleavage. Two points at $(B D E, S Y)=(0,0)$ and $(10,1)$ are added for fitting purposes. The data is fit to a sigmoidal function of the form described in Equation 13 according to prior implementations of the method.[19, 24, 25]

$$
y=\frac{a}{1+\exp \left(\frac{-\left(x-x_{0}\right)}{b}\right)}
$$

where $a, b$, and $x_{0}$ are fit parameters. By taking the derivative of the sigmoid, one obtains an approximation of the internal energy distributions of the ions (equation 14). Select sigmoidal functions and their corresponding derivatives fit with Boltzmann distributions for $p$-OMe are shown in Figure S16 and S17, respectively.

$$
\frac{d y}{d x}=\left(\frac{a}{b}\right) \cdot\left(\frac{\exp \left(\frac{-\left(x-x_{0}\right)}{b}\right)}{1+\left(\exp \left(\frac{-\left(x-x_{0}\right)}{b}\right)\right)^{2}}\right)
$$

$T_{\text {eff }}$ is determined at a given DP by fitting Boltzmann distributions (Equation 6) for the BP ions used such that their average internal energy matches that of the sigmoid derivative. This is shown for the $p$-OMe derivative at DP $=100 \mathrm{~V}$ in Figure S18. As each BP derivative contains a unique $\mathrm{R}$ group, the difference in the rovibrational density of states translates to a unique $T_{\text {eff }}$ experienced for each BP ion. The SY and corresponding $T_{\text {eff }}$ of each BP ion is shown in Figure 7. The $p$-Cl BP derivative could not be treated with this method due to a fragmentation channel that resulted in loss of the $\mathrm{Cl}$ moiety; the resulting fragment could not be distinguished as originating from the either ${ }^{35} \mathrm{Cl}$ or ${ }^{37} \mathrm{Cl}$ parent isotopologues.

At the default setting of DP $=100 \mathrm{~V}$, with no influence from the $S \mathrm{~V}$ and $T_{\text {bath }}=373 \mathrm{~K}$, the maximum $T_{\text {eff }}$ for the $\mathrm{BP}$ ion series varies from $768 \pm 26 \mathrm{~K}(p-\mathrm{OMe})$ to $877 \pm 24 \mathrm{~K}(p-\mathrm{H} \mathrm{BP})$ in the QJet region. 
As mentioned previously, the degree of ion heating induced by the DP is proportional to intrinsic ion properties (i.e., mass, $\mathrm{N}_{\mathrm{DoF}}, \mathrm{CCS}$ ). However, one must consider the ramifications of field induced heating at significantly lower pressure, where collisional cooling is inefficient. The approximate initial pressure in the QJet region is 2 Torr, which drops off significantly as the ion travels further into the mass spectrometer (operating at $c a .7 \mathrm{mTorr}$ ). With $\mathrm{N}_{2}$ and $T_{\text {bath }}=373 \mathrm{~K}$, the mean free path is $c a .0 .04 \mathrm{~cm}$ at 2 Torr and $c a$. $10.3 \mathrm{~cm}$ at $7 \mathrm{mTorr}$. Since the total length of the QJet and Q0 regions being $15 \mathrm{~cm}$, this translates to fewer than 400 collisions between residual buffer gas and the analyte. This is in direct contrast to the DMS cell at atmospheric pressure where millions of collisions occur during the $c a .6$ ms transit time. In the absence of sufficient collisions, a linear relationship exists between $T_{\text {eff }}$ of the BP derivatives and the product of the ion mass and its $3 N$ degrees of freedom (i.e., negligible dependence on CCS). These are plotted in Figure S19, where correlation coefficients of $R^{2}=0.98,0.98$, and 0.96 are found at $D P=60,120$, and $180 \mathrm{~V}$. Correlations for DP heating that consider inverse CCS dependence yield an average $\mathrm{R}^{2}$ of 0.87 . Linear fit parameters for heating at all DPs sampled can be found in the supporting information. 


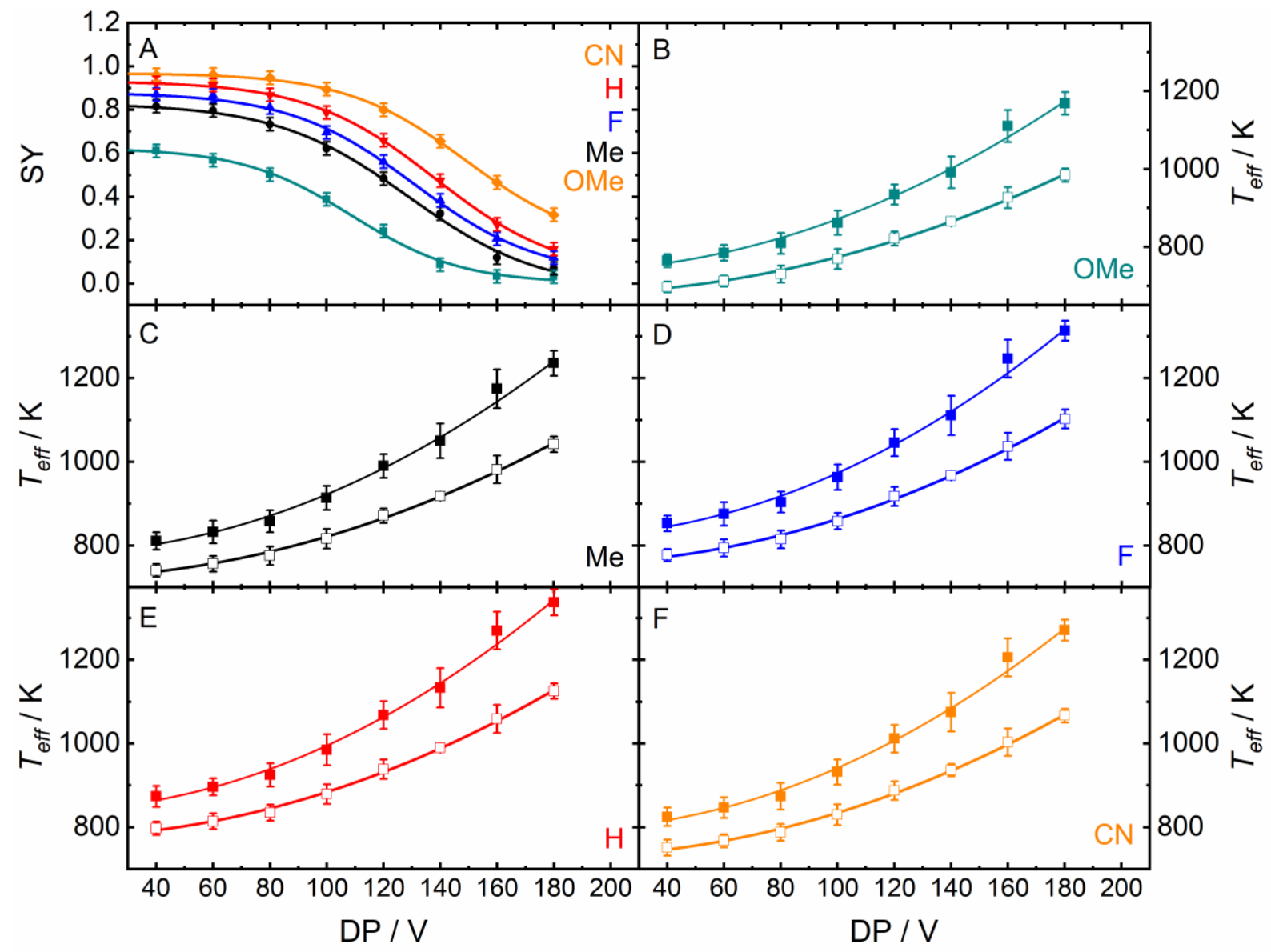

Figure 7. (A) Survival yields and effective ion temperatures for the (B) $p$-OMe, (C) $p-\mathrm{Me}$, (D) $p-\mathrm{F}$, (E) $p-H$, and $(\mathbf{F}) p$-CN BP ions as a function of DP. Curves correspond to a quadratic fit of the form $y=a x^{2}+b$ ( $\left.T_{\text {bath }}=373 \mathrm{~K}\right) . T_{\text {eff }}$ is determined by incorporating the kinetic shift (solid squares) or neglecting its effect (open squares).

The methodology employed to determine $T_{\text {eff }}$ in the QJet region does not consider the fact that BP ions have a finite amount of time to dissociate upon activation. To account for the finite time spent in the QJet region, sigmoidal curves can be generated for $S Y$ versus the appearance energy $\left(E_{a p p}\right)$, where $E_{a p p}$ is the energy necessary to fragment the BP ion at an observable rate. $E_{a p p}$ is the sum of the critical energy, $E_{0}$ (i.e., the zero-point dissociation energy of the ion) and the kinetic shift, $E_{k s}$, which is the excess energy required to observe dissociation on the experimental timescale.[27,67] The timescale for ion activation 
in the QJet region is approximately $4 \mu \mathrm{s}$. However, fragmentation can occur along the length of the QJet and $\mathrm{Q} 0$ regions as well as throughout the mass spectrometer en route to the detector. Should fragmentation occur between $\mathrm{Q} 1$ and $\mathrm{Q} 3$, an increase in the respective $\mathrm{M}^{+} / \mathrm{F}^{+} \mathrm{MRM}$ transitions would be observed; since such an increase was not detected, it was assumed that dissociation predominantly occurs in the QJet and Q0 region. A schematic of the mass spectrometer highlighting the locations of the QJet, Q0, Q1, and Q3 is provided in Figure S20. Thus, the timescale for dissociation in this region is dictated by the time it takes the ion to transit the QJet region and $\mathrm{Q} 0$, which is approximately $6 \pm 2 \mathrm{~ms}\left(\tau_{D P}\right)$. A description of this determination is provided in the supporting information. $E_{a p p}$ values are then determined from $\mathrm{k}\left(\mathrm{E}_{\mathrm{app}}\right)=\tau_{D P^{-1}}$ using Figure $\mathrm{S} 1$. This yields the following average $E_{a p p}$ values: $2.23(p-\mathrm{OMe})$, $2.84(p-\mathrm{Me}), 2.93(p-\mathrm{Cl}), 2.95(p-\mathrm{F}), 3.09(p-\mathrm{H})$, and $3.72 \mathrm{eV}(p-\mathrm{CN})$. Accounting for the kinetic shift raises temperatures significantly, as seen in Figure 7. It should be noted that there will be competition between some collision induced fragmentation and cooling in this region, so temperatures are likely cooler than those predicted by the kinetic shift.

\section{In-source and external influences on $T_{\text {eff }}$}

In previous works that used BP ions to evaluate $T_{\text {eff }}$ for in-source heating, it was shown that ESI capillary temperature has an effect on the degree of heating imparted to the ions.[24] In cases where ions thermalize to $T_{\text {bath }}$, a source temperature that is below $T_{\text {bath }}$ will not affect the SY at SV $=0 \mathrm{~V}$. In cases where ions are not thermalized to $T_{b a t h}$, it should be possible to change SY by varying source temperature. Figure S21 shows the source temperature dependence of the SY for the most weakly-bound BP derivative ( $p$-OMe) at $T_{\text {bath }}=373 \mathrm{~K}$ and SV $=0 \mathrm{~V}$; changes in SY as a function of increasing source temperature are within statistical error. This shows that, although the ESI process activates ions resulting in in-source fragmentation, the ions produced by ESI are quickly thermalized to $T_{\text {bath }}$ en route to the DMS cell. This result accords with other studies of ESI source temperatures assessed using BP ions.[21, 46, 66, 68] 
It has also been suggested that the ESI solvent impacts the observed $T_{\text {eff }}$ in experiments.[23, 26] Despite the fact that this phenomenon stems from droplet evaporation during the ESI process, the relative reactivity and stability of the benzylpyridinium thermometer ions must be considered owing to the relative electrophilicity of the benzyl position. In the presence of protic ESI solvents, hydrolysis to yield the benzyl alcohol or benzyl ether is likely. Investigation of four solvent mixtures of $\mathrm{MeCN}$ with either $\mathrm{MeOH}$ or $\mathrm{H}_{2} \mathrm{O}$ showed minor differences in the SY for the $p$-OMe BP ion in experiments conducted on the same day (see Figure S22). The SYs for species produced from methanol containing ESI solvents were marginally greater than those from mixtures containing water. However, SYs in ESI mixtures containing water exhibited much larger variance between measurements, which can be predominantly attributed to formation of the substituted benzyl alcohol $(m / z 139)$ during the ESI process. Analysis of the ionograms indicates that $15.1 \pm 2.0 \%$ of the total peak area corresponds to formation of the benzyl alcohol when a 9:1 $\mathrm{H}_{2} \mathrm{O} / \mathrm{MeCN}$ solution is employed, and that this value is reduced to $7.6 \pm 0.4 \%$ when a $1: 1 \mathrm{H}_{2} \mathrm{O} / \mathrm{MeCN}$ used. Negligible amounts of the methyl benzyl ether $(\mathrm{m} / \mathrm{z} 153)$ was produced from ESI mixtures containing methanol $(<2 \%)$.

In the evaluations of in-source and external influences on $T_{\text {eff, }}$ instrumental parameters and components (i.e., ESI capillary, TurboSpray source, DMS cell and plates) were fixed. The extent of ion activation using three different TurboSpray sources was also examined. Figure S23 shows differences in SY of the $p$-OMe derivative as a function of TurboSpray sources operating under identical conditions. These combined results are indicative of the absolute uncertainty related to a SY measurement. Day-to-day drifts in experimental SY can originate from more variable conditions, which may include the state of the vacuum system, trace humidity present in the $\mathrm{N}_{2}$ carrier gas, and the cleanliness of the ESI capillary and DMS cell. It is therefore necessary to characterize SY on subsequent days to evaluate the uncertainty associated with any single SY determination. 


\section{Conclusions}

The extent of ion heating during DMS experiments is evaluated experimentally for the first time by using the survival yields of benzylpyridinium thermometer ions to determine effective temperatures using an RRKM-based approach. The extent of ion activation was found to depend linearly on the ion density of states and collision frequency. Our analysis indicates extensive heating induced by the SV waveform at high peak-to-peak voltage, with effective ion temperatures ranging from $664 \pm 9 \mathrm{~K}$ (p-OMe) to $759 \pm 17 \mathrm{~K}$ $(p-\mathrm{H})$ for the benzylpyridinium derivatives at $\mathrm{SV}=4400 \mathrm{~V}$ and $T_{\text {bath }}=450 \mathrm{~K}$. The decrease in SY with increasing SV while minimizing post-DMS potentials unambiguously identifies that the SV is responsible for ion activation and fragmentation in the DMS cell. Similar analysis for the post-DMS region indicate substantial ion heating exceeding that observed in the DMS cell owing to the substantially larger $E / N$ ratio.

By characterizing ion temperatures and establishing a correlation between $T_{\text {eff }}$ and inherent ion properties (viz. mass, number of degrees of freedom, and CCS), this work provides a step towards elucidating the fundamental principles that underpin the phenomenon of differential ion mobility. Whether this linear trend can be generalized to all molecular classes is an open question. Nevertheless, the fact that $T_{\text {eff }}$ significantly exceeds $T_{\text {bath }}$ at high field has important bearing on the interpretation of DMS data related to ion structure. On the one hand, the dynamic temperature conditions and relatively high peak temperatures complicate the evaluation of CCS by DMS because the high temperature and pressure conditions have the potential to induce structural rearrangements of the analytes. While dynamic ion CCSs associated with ion microsolvation are relatively well established in the context of DMS, [62] dynamic analyte structural changes (e.g., protein folding and unfolding) have received less attention.[15, 69] On the other hand, a deeper understanding of the well-defined and controlled conditions of the DMS environment, and in particular how this affects ion temperature, presents the opportunity to study dynamic phenomena like isomerization and ion-solvent clustering. Ultimately, the determination of CCSs from DMS experiments relies on gauging the dynamic effective temperature of the ion in question during 
the SV duty cycle. This temperature range must then be correlated to the temperature-dependent evolution of ion structure to create a picture of the dynamic CCS of the ion. [70, 71] As progress is made in this regard, the direct correlation of DMS measurements to molecular structure may become possible.

\section{Acknowledgements}

The authors would like to further acknowledge Bruce Collings (SCIEX) who contributed SIMION simulations, Prof. Terry McMahon (University of Waterloo) for helpful discussions, and the highperformance computing support from Compute Canada. We additionally thank the reviewers of this manuscript for their collective insights. WSH would like to acknowledge the financial support provided by the Natural Sciences and Engineering Research Council (NSERC) of Canada, the Ontario Centres of Excellence in the form of a VIP-II grant, as well as the government of Ontario for an Ontario Early Researcher Award. $\mathrm{Cl}$ acknowledges financial support from the government of Ontario for an Ontario Graduate Scholarship.

\section{Conflicts of Interest}

The authors declare that there are no conflicts of interest.

\section{Supporting Information}

Supplementary Figures S1-S23 and Tables S1-S2 as described in the manuscript, calculated bond dissociation energies, all function fit parameters, QJet/Q0 transit times, collision cross sections, calculated 
vibrational frequencies, rotational constants, ion geometries, and author contributions are available in the supporting information accompanying this manuscript.

\section{References}

1. Collins, D.C., Lee, M.L.: Developments in ion mobility spectrometry-mass spectrometry. Anal. Bioanal. Chem. 372, 66-73 (2002). doi:10.1007/s00216-001-1195-5

2. Lapthorn, C., Pullen, F., Chowdhry, B.Z.: Ion mobility spectrometry-mass spectrometry (IMS-MS) of small molecules: Separating and assigning structures to ions. Mass Spectrom. Rev. 32, 43-71 (2013). doi:10.1002/mas

3. Paglia, G., Astarita, G.: Metabolomics and lipidomics using traveling-wave ion mobility mass spectrometry. Nat. Protoc. 12, 797-813 (2017). doi:10.1038/nprot.2017.013

4. Verkouteren, J.R., Staymates, J.L.: Reliability of ion mobility spectrometry for qualitative analysis of complex, multicomponent illicit drug samples. Forensic Sci. Int. 206, 190-196 (2011). doi:10.1016/j.forsciint.2010.08.005

5. Cumeras, R., Figueras, E., Davis, C.E., Baumbach, J.I., Gràcia, I.: Review on lon Mobility Spectrometry. Part 1: Current instrumentation. Analyst. 140, 1376-1390 (2015). doi:10.1039/c4an01100g

6. Lanucara, F., Holman, S.W., Gray, C.J., Eyers, C.E.: The power of ion mobility-mass spectrometry for structural characterization and the study of conformational dynamics. Nat. Chem. 6, 281-294 (2014). doi:10.1038/nchem.1889

7. Cajka, T., Fiehn, O.: Toward Merging Untargeted and Targeted Methods in Mass Spectrometry- 
Based Metabolomics and Lipidomics. Anal. Chem. 88, 524-545 (2016). doi:10.1021/acs.analchem.5b04491

8. Mason, E.A., McDaniel, E.W.: Transport properties of ions in gases. John Wiley and Sons, New York (1988)

9. Shvartsburg, A.A., Smith, R.D.: Fundamentals of traveling wave ion mobility spectrometry. Anal. Chem. 80, 9689-9699 (2008). doi:10.1021/ac8016295

10. Schneider, B.B., Covey, T.R., Coy, S.L., Krylov, E. V., Nazarov, E.G.: Planar differential mobility spectrometer as a pre-filter for atmospheric pressure ionization mass spectrometry. Int. J. Mass Spectrom. 298, 45-54 (2010). doi:10.1016/j.ijms.2010.01.006

11. Shvartsburg, A.A.: Differential ion mobility spectrometry: nonlinear ion transport and fundamentals of FAIMS. CRC Press, Boca Raton (2009)

12. Guevremont, R.: High-field asymmetric waveform ion mobility spectrometry: A new tool for mass spectrometry. J. Chromatogr. A. 1058, 3-19 (2004). doi:10.1016/j.chroma.2004.08.119

13. Morsa, D., Gabelica, V., De Pauw, E.: Fragmentation and isomerization due to field heating in traveling wave ion mobility spectrometry. J. Am. Soc. Mass Spectrom. 25, 1384-1393 (2014). doi:10.1007/s13361-014-0909-9

14. Merenbloom, S.I., Flick, T.G., Williams, E.R.: How hot are your ions in TWAVE ion mobility spectrometry? J. Am. Soc. Mass Spectrom. 23, 553-562 (2012). doi:10.1007/s13361-011-0313-7

15. Shvartsburg, A.A., Li, F., Tang, K., Smith, R.D.: Distortion of ion structures by field asymmetric waveform ion mobility spectrometry. Anal. Chem. 79, 1523-1528 (2007). doi:10.1021/ac061306c

16. Revercomb, H.E., Mason, E.A.: Theory of Plasma Chromatography/Gaseous Electrophoresis. A 
Review. Anal. Chem. 47, 970-983 (1975). doi:10.1021/ac60357a043

17. de Pauw, E., Pelzer, G., Marien, J., Natalis, P.: Internal Energy Distribution of Ions Emitted in Secondary Ion Mass Spectrometry. Springer Proceedings in Physics, vol 9. Springer, Berlin, Heidelberg (1986)

18. Derwa, F., de Pauw, E., Natalis, P.: New Basis for a Method for the Estimation of Secondary lon Internal Energy Distribution in "Soft" Ionization Techniques. Org. Mass Spectrom. 26, 117-118 (1991)

19. Carpenter, J.E., McNary, C.P., Furin, A., Sweeney, A.F., Armentrout, P.B.: How Hot are Your lons Really? A Threshold Collision-Induced Dissociation Study of Substituted Benzylpyridinium “Thermometer" Ions. J. Am. Soc. Mass Spectrom. 28, 1876-1888 (2017). doi:10.1007/s13361-017$1693-0$

20. Gatineau, D., Memboeuf, A., Milet, A., Cole, R.B., Dossmann, H., Gimbert, Y., Lesage, D.: Experimental bond dissociation energies of benzylpyridinium thermometer ions determined by threshold-CID and RRKM modeling. Int. J. Mass Spectrom. 417, 69-75 (2017). doi:10.1016/j.ijms.2017.03.002

21. Debord, J.D., Verkhoturov, S. V., Perez, L.M., North, S.W., Hall, M.B., Schweikert, E.A.: Measuring the internal energies of species emitted from hypervelocity nanoprojectile impacts on surfaces using recalibrated benzylpyridinium probe ions. J. Chem. Phys. 138, 214301-1-214301-9 (2013). doi:10.1063/1.4807602

22. Barylyuk, K. V., Chingin, K., Balabin, R.M., Zenobi, R.: Fragmentation of Benzylpyridinium "Thermometer" Ions and Its Effect on the Accuracy of Internal Energy Calibration. J. Am. Soc. Mass Spectrom. 21, 172-177 (2010). doi:10.1016/j.jasms.2009.09.023 
23. Gabellca, V., De Pauw, E.: Internal energy and fragmentation of ions produced in electrospray sources. Mass Spectrom. Rev. 24, 566-587 (2005). doi:10.1002/mas.20027

24. Gabelica, V., De Pauw, E., Karas, M.: Influence of the capillary temperature and the source pressure on the internal energy distribution of electrosprayed ions. Int. J. Mass Spectrom. 231, 189-195 (2004). doi:10.1016/j.ijms.2003.10.010

25. Drahos, L., Heeren, R.M.A., Collette, C., De Pauw, E., Vekey, K.: Thermal energy distribution observed in electrospray ionization. J. Mass Spectrom. 34, 1373-1379 (1999). doi:10.1002/(SICI)1096-9888(199912)34:12<1373::AID-JMS907>3.0.CO;2-\#

26. Collette, C., De Pauw, E.: Calibration of the internal energy distribution of ions produced by electrospray. Rapid Commun. Mass Spectrom. 12, 165-170 (1998). doi:10.1002/(SICI)10970231(19980227)12:4<165::AID-RCM140>3.0.CO;2-1

27. Collette, C., Drahos, L., De Pauw, E., Vékey, K.: Comparison of the internal energy distributions of ions produced by different electrospray sources. Rapid Commun. Mass Spectrom. 12, 1673-1678 (1998). doi:10.1002/(SICI)1097-0231(19981130)12:22<1673::AID-RCM385>3.0.CO;2-A

28. Luo, G., Marginean, I., Vertes, A.: Internal energy of ions generated by matrix-assisted laser desorption/ionization. Anal. Chem. 74, 6185-6190 (2002). doi:10.1021/ac020339z

29. Flanigan, P.M., Shi, F., Archer, J.J., Levis, R.J.: Internal energy deposition for low energy, femtosecond laser vaporization and nanospray post-ionization mass spectrometry using thermometer ions. J. Am. Soc. Mass Spectrom. 26, 716-724 (2015). doi:10.1007/s13361-015-10816

30. Stephens, E.R., Dumlao, M., Xiao, D., Zhang, D., Donald, W.A.: Benzylammonium Thermometer Ions: Internal Energies of Ions Formed by Low Temperature Plasma and Atmospheric Pressure 
Chemical Ionization. J. Am. Soc. Mass Spectrom. 26, 2081-2084 (2015). doi:10.1007/s13361-015$1272-1$

31. Rahrt, R., Auth, T., Demireva, M., Armentrout, P.B., Koszinowski, K.: Benzhydrylpyridinium lons: A New Class of Thermometer Ions for the Characterization of Electrospray-Ionization Mass Spectrometers. Anal. Chem. 91, 11703-11711 (2019). doi:10.1021/acs.analchem.9b02257

32. Morsa, D., Gabelica, V., De Pauw, E.: Effective temperature of ions in traveling wave ion mobility spectrometry. Anal. Chem. 83, 5775-5782 (2011). doi:10.1021/ac201509p

33. Santiago, B.G., Campbell, M.T., Glish, G.L.: Variables Affecting the Internal Energy of Peptide lons During Separation by Differential Ion Mobility Spectrometry. J. Am. Soc. Mass Spectrom. 28, 21602169 (2017). doi:10.1007/s13361-017-1726-8

34. Schneider, B.B., Covey, T.R., Coy, S.L., Krylov, E. V, Nazarov, E.G.: Planar differential mobility spectrometer as a pre-filter for atmospheric pressure ionization mass spectrometry. Int. J. Mass Spectrom. 298, 45-54 (2010). doi:10.1016/j.ijms.2010.01.006

35. Schneider, B.B., Nazarov, E.G., Londry, F., Vouros, P., Covey, T.R.: Differential Mobility Spectrometry/Mass Spectrometry History, Theory, Design Optimization, Simulations, and Applications. Mass Spectrom. Rev. 35, 687-737 (2016). doi:10.1002/mas.21453

36. Liu, C., Le Blanc, J.C.Y., Shields, J., Janiszewski, J.S., leritano, C., Ye, G.F., Hawes, G.F., Hopkins, W.S., Campbell, J.L.: Using differential mobility spectrometry to measure ion solvation: an examination of the roles of solvents and ionic structures in separating quinoline-based drugs. Analyst. 14, 68976903 (2015). doi:10.1039/C5AN00842E

37. Liu, C., Yves Le Blanc, J.C., Schneider, B.B., Shields, J., Federico, J.J., Zhang, H., Stroh, J.G., Kauffman, G.W., Kung, D.W., leritano, C., Shepherdson, E., Verbuyst, M., Melo, L., Hasan, M., Naser, D., 
Janiszewski, J.S., Hopkins, W.S., Campbell, J.L.: Assessing Physicochemical Properties of Drug Molecules via Microsolvation Measurements with Differential Mobility Spectrometry. ACS Cent. Sci. 3, 101-109 (2017). doi:10.1021/acscentsci.6b00297

38. Marcus, R.A.: Unimolecular Dissociations and Free Radical Recomibation Reactions. J. Chem. Phys. 20, 359-364 (1952)

39. Stein, S.E., Rabinovitch, B.S.: Accurate evaluation of internal energy level sums and densities including anharmonic oscillators and hindered rotors. J. Chem. Phys. 58, 2438-2445 (1973). doi:10.1063/1.1679522

40. Beyer, T., Swinehart, D.F.: Number of multiply-restricted partitions. Commun. Assocation Comput. Mach. 16, 379 (1973). doi:10.1145/362248.362275

41. J. R. Barker, T. L. Nguyen, J. F. Stanton, C. Aieta, M. Ceotto, F. Gabas, T. J. D. Kumar, C. G. L. Li, L. L. Lohr, A. Maranzana, N. F. Ortiz, J. M. Preses, J. M. Simmie, J. A. Sonk, and P. J. Stimac; MultiWell2019 Software Suite; J. R. Barker, University of Michigan, Ann Arbor, Michigan, USA, 2019; http://clasp-research.engin.umich.edu/multiwell/.

42. Barker, J.R.: Multiple-well, multiple-path unimolecular reaction systems. I. MultiWell computer program suite. Int. J. Chem. Kinet. 33, 232-245 (2001). doi:10.1002/kin.1017

43. Barker, J.R.: Energy Transfer in Master Equation Simulations: A New Approach. Int. J. Chem. Kinet. 41, 748-763 (2009). doi:10.1002/kin

44. Rodgers, M.T., Ervin, K.M., Armentrout, P.B.: Statistical modeling of sequential collision-induced dissociation thresholds. J. Chem. Phys. 126, 4499-4508 (1997). doi:10.1063/1.2741550

45. Rodgers, M.T., Armentrout, P.B.: Statistical modeling of competitive threshold collision-induced dissociation. J. Chem. Phys. 109, 1787-1800 (1998). doi:10.1063/1.476754 
46. Morsa, D., Gabelica, V., Rosu, F., Oomens, J., De Pauw, E.: Dissociation pathways of benzylpyridinium "thermometer" ions depend on the activation regime: An IRMPD spectroscopy study. J. Phys. Chem. Lett. 5, 3787-3791 (2014). doi:10.1021/jz501903b

47. Waage, E. V., Rabinovitch, B.S.: Centrifugal effects in reaction rate theory. Chem. Rev. 70, 377-387 (1970). doi:10.1021/cr60265a004

48. M. J. Frisch, G. W. Trucks, H. B. Schlegel, G. E. Scuseria, M. A. Robb, J. R. Cheeseman, G. Scalmani, B. V. Barone, G. Mennucci and A. Petersson, Gaussian 16, Revision B.01, Gaussian, Inc., Wallingford CT, 2016.

49. Becke, A.D.: Density-Functional Exchange-Energy Approximation with Corrects AsymptoticBehavior. Phys. Rev. A. 38, 3098-3100 (1988). doi:10.1103/PhysRevA.38.3098

50. Lee, C., Yang, W., Parr, R.G.: Development of the Colle-Salvetti correlation-energy formula into a functional of the electron density. Phys. Rev. B. 37, 785-789 (1988). doi:10.1103/PhysRevB.37.785

51. Chai, J., Head-Gordon, M.: Long-range corrected hybrid density functionals with improved dispersion corrections. Phys. Chem. Chem. Phys. 10, 6615-6620 (2008). doi:10.1021/ct300715s

52. Andersson, M.P., Uvdal, P.: New scale factors for harmonic vibrational frequencies using the B3LYP density functional method with the triple- $\zeta$ basis Set 6-311+G(d,p). J. Phys. Chem. A. 109, 29372941 (2005). doi:10.1021/jp045733a

53. Singh, U.C., Kollman, P.A.: An approach to computing electrostatic charges for molecules. J. Comput. Chem. 5, 129-145 (1984). doi:10.1002/jcc.540050204

54. Besler, B.H., Merz, K.M., Kollman, P.A.: Atomic charges derived from semiempirical methods. J. Comput. Chem. 11, 431-439 (1990). doi:10.1002/jcc.540110404 
55. Ieritano, C., Crouse, J., Campbell, J.L., Hopkins, S.: A parallelized molecular collision cross section package with optimized accuracy and efficiency. Analyst. 144, 1660-1670 (2019). doi:10.1039/C8AN02150C

56. Siems, W.F., Viehland, L.A., Hill, H.H.: Correcting the fundamental ion mobility equation for field effects. Analyst. 141, 6396-6407 (2016). doi:10.1039/c6an01353h

57. Siems, W.F., Viehland, L.A., Hill, H.H.: Improved momentum-transfer theory for ion mobility. 1. Derivation of the fundamental equation. Anal. Chem. 84, 9782-9791 (2012). doi:10.1021/ac301779s

58. Waldman, M., Mason, E.A.: Generalized Einstein relations from a three-temperature theory of gaseous ion transport. Chem. Phys. 58, 121-144 (1981). doi:10.1016/0301-0104(81)80050-X

59. Donor, M.T., Mroz, A.M., Prell, J.S.: Experimental and theoretical investigation of overall energy deposition in surface-induced unfolding of protein ions. Chem. Sci. 10, 4097-4106 (2019). doi:10.1039/C9SC00644C

60. Haack, A., Crouse, J., Schlüter, F.-J., Benter, T., Hopkins, W.S.: A First Principles Model of Differential Ion Mobility: The Effect of Ion-Solvent Clustering. J. Am. Soc. Mass Spectrom. (2019). doi:10.1007/s13361-019-02340-1

61. Wyttenbach, T., Von Helden, G., Batka, J.J., Carlat, D., Bowers, M.T.: Effect of the long-range potential on ion mobility measurements. J. Am. Soc. Mass Spectrom. 8, 275-282 (1997). doi:10.1016/S1044-0305(96)00236-X

62. Hopkins, W.S.: Dynamic Clustering and lon Microsolvation. In: Donald, W.A., Prell, J.S., and Barcelò, D. (eds.) Advances in Ion Mobilty-Mass Spectrometry: Fundamentals Instrumentation, and Applications. pp. 83-118. Elsevier (2019) 
63. Schneider, B.B., Londry, F., Nazarov, E.G., Kang, Y., Covey, T.R.: Maximizing Ion Transmission in Differential Mobility Spectrometry. J. Am. Soc. Mass Spectrom. 28, 2151-2159 (2017). doi:10.1007/s13361-017-1727-7

64. Lai, R., Dodds, E.D., Li, H.: Molecular dynamics simulation of ion mobility in gases. J. Chem. Phys. 148, (2018). doi:10.1063/1.4998955

65. Naban-Maillet, J., Lesage, D., Bossée, A., Gimbert, Y., Sztáray, J., Vékey, K., Tabet, J.C.: Internal energy distribution in electrospray ionization. J. Mass Spectrom. 40, 1-8 (2005). doi:10.1002/jms.773

66. Barylyuk, K. V., Chingin, K., Balabin, R.M., Zenobi, R.: Fragmentation of Benzylpyridinium "Thermometer" Ions and Its Effect on the Accuracy of Internal Energy Calibration. J. Am. Soc. Mass Spectrom. 21, 172-177 (2010). doi:10.1016/j.jasms.2009.09.023

67. Vékey, K.: Internal energy effects in mass spectrometry. J. Mass Spectrom. 31, 445-463 (1996). doi:10.1002/(SICI)1096-9888(199605)31:5<445::AID-JMS354>3.0.CO;2-G

68. Zins, E.L., Pepe, C., Rondeau, D., Rochut, S., Galland, N., Tabet, J.C.: Theoretical and experimental study of tropylium formation from substituted benzylpyridinium species. J. Mass Spectrom. 44, 12-17 (2009). doi:10.1002/jms.1461

69. Zhu, S., Campbell, J.L., Chernushevich, I., Le Blanc, J.C.Y., Wilson, D.J.: Differential Mobility Spectrometry-Hydrogen Deuterium Exchange (DMS-HDX) as a Probe of Protein Conformation in Solution. J. Am. Soc. Mass Spectrom. 27, 991-999 (2016). doi:10.1007/s13361-016-1364-6

70. leritano, C., Campbell, J.L., Hopkins, W.S.: Unravelling the factors that drive separation in differential mobility spectrometry: A case study of regioisomeric phosphatidylcholine adducts. Int. J. Mass Spectrom. 444, (2019). doi:10.1016/j.ijms.2019.116182 
71. Zhou, C., leritano, C., Hopkins, W.S.: Augmenting Basin-Hopping With Techniques From Unsupervised Machine Learning : Applications in Spectroscopy and Ion Mobility. Front. Chem. 7, 519 (2019). doi:10.3389/fchem.2019.00519 